\title{
Circular RNA _0015278 inhibits the progression of non-small cell lung cancer through regulating the microRNA 1278/SOCS6 gene axis
}

\author{
Yiwang Ye ${ }^{1}$, Xuan Wu ${ }^{2}$, Feihu Long ${ }^{1}$, Wei Yue ${ }^{1}$, Da Wu ${ }^{1}$, Yuancai Xie ${ }^{1}$ \\ ${ }^{1}$ Department of Thoracic Surgery, Peking University Shenzhen Hospital, Shenzhen, China; ${ }^{2}$ Department of Oncology, Peking University Shenzhen \\ Hospital, Shenzhen, China \\ Contributions: (I) Conception and design: Y Ye, Y Xie; (II) Administrative support: X Wu; (III) Provision of study materials or patients: Y Ye, F Long, \\ W Yue, D Wu; (IV) Collection and assembly of data: All authors; (V) Data analysis and interpretation: Y Ye, X Wu, W Yue, D Wu, Y Xie; (VI) \\ Manuscript writing: All authors; (VII) Final approval of manuscript: All authors. \\ Correspondence to: Yuancai Xie. Department of Thoracic Surgery, Peking University Shenzhen Hospital, No.1120 Lianhua Road, Futian District, \\ Shenzhen 518036, China. Email: Xieyuancai2005@126.com.
}

Background: Non-small cell lung cancer (NSCLC) is one of the most lethal malignancies worldwide. Deepening understanding of the pathogenesis of NSCLC is quite important for its treatment. Circular (circ) RNA_0015278 has been found to be downregulated in NSCLC, but its role in NSCLC and the underlying regulatory mechanism is unknown.

Methods: Circ_0015278, microRNA (miR)-1278 and SOCS6 were analyzed with real-time quantitative reverse transcription polymerase chain reaction (qRT-PCR) or western blot. Cell Counting Kit-8 (CCK-8) and 5-ethynyl-2'-deoxyuridine (EdU) staining were used to evaluate cell proliferation. The colony forming capacity and invasion of NSCLC cells were assessed with colony formation and transwell assays, respectively. The interaction among circ_0015278, miR-1278, and SOCS6 was evaluated using luciferase, receptor interacting protein (RIP), and RNA-pull down assays. Cell apoptosis was analyzed using flow cytometry. A subcutaneous NSCLC xenograft mouse model was established for evaluating circ_0015278-mediated effects on the growth of NSCLC in vivo.

Results: Circ_0015278 was downregulated in NSCLC tissues and cells, and its reduced expression indicated poor prognosis. Overexpression of circ_0015278 restrained the proliferation, colony formation, invasion, and epithelial-mesenchymal transition (EMT) of NSCLC cells and induced NSCLC cell apoptosis. Moreover, overexpression of circ_0015278 inhibited the growth of NSCLC in vivo. Mechanically, circ_0015278 acted as an miR-1278 sponge to reduce its quantity, and miR-1278 targeted SOCS6 to inhibit its expression in NSCLC cells. Circ_0015278 promoted SOCS6 expression by sponging miR-1,278 in NSCLC cells. Overexpression of circ_0015278 attenuated the malignant phenotypes of NSCLC through sponging miR-1278 and consequently promoting SOCS6 expression.

Conclusions: We demonstrated for the first time that circ_0015278 attenuated the progression of NSCLC via targeting the miR-1278/SOCS6 axis, which provides potential diagnostic biomarkers and therapeutic targets.

Keywords: Circular RNA_0015278; migration; invasion; microRNA 1278; SOCS6

Submitted Jun 16, 2021. Accepted for publication Jul 23, 2021.

doi: $10.21037 / \mathrm{atm}-21-3456$

View this article at: https://dx.doi.org/10.21037/atm-21-3456 


\section{Introduction}

Lung cancer, originating in the lung, is one of the most common types of malignancies and the leading cause of cancer-related deaths globally. It can be divided into two major groups, nonsmall cell lung cancer (NSCLC) and small cell lung cancer (SCLC) $(1,2)$. NSCLC is the most common lung cancer, accounting for about $85 \%$ of all cases (3). Unfortunately, only a small number of patients with NSCLC (21\%) are diagnosed at stage I due to no obvious symptoms, and most patients (61\%) are diagnosed at the advanced stages (stage III or IV), leading to the huge decline of 5 -year survival from $57 \%$ (stage I) to 4\% (stage IV) (4). Although increasing advances have been made recently in NSCLC therapies, the high malignancy and metastasis potential of NSCLC leads to therapeutic failure and very poor prognosis (5). Therefore, elucidating the pathogenesis and progression of NSCLC is crucial for seeking early diagnostic biomarkers and therapeutic targets.

As one of the non-coding RNAs, circular (circ) RNAs form continuous loops through covalently linking the $5^{\prime}$ and $3^{\prime}$ termini and play important roles in various physiological and pathological conditions (6,7). Intriguingly, increasing evidence has shown that circRNAs can function as oncogenes or tumor suppressors in NSCLC (8). Jiang and colleagues found that hsa_circ_0007385 expression was elevated in NSCLC tissues, and knockdown of hsa circ_0007385 restrained NSCLC cell proliferation, migration, and invasion in vitro and restrained its growth in vivo (9). Circular RNA Forkhead box O3 (circRNAFOXO3) was reported to be reduced in NSCLC cells, and its overexpression clearly repressed NSCLC cell migration and invasion (10). Circular RNA_0015278 (circ_0015278), also known as circ_100395, is downregulated in NSCLC tissues (11), and overexpression of circ_0015278 suppressed the progression of lung cancer (12). However, more studies are required to elucidate the role of circ_0015278 and the underlying mechanisms in NSCLC.

CircRNAs have been well acknowledged to exert oncogenic or anti-tumor activity via acting as competitive endogenous RNAs (ceRNAs) to sponge microRNAs (miRNAs), thereby relieving miRNA-mediated suppressive effects on downstream targets, in various human cancers including NSCLC $(13,14)$. Two independent studies have shown that circ_0015278 targeted microRNA (miR)1228 to exert its function in ovarian and lung cancers (15) Micro RNAs also serve as vital modulators in cancers (16). MicroRNA-1278 (MiR-1278) was reported to be downregulated in nasopharyngeal carcinoma and colorectal cancer and to exert anti-tumor activity in both cancers (17). However, its oncogenic activity and roles in NSCLC to our knowledge have not been reported. In addition, the interaction between circ_0015278 and miR-1278 is unknown.

Suppressor of cytokine signaling 6 (SOCS6) exerts antitumor effects in various human cancers including prostate cancer (18), esophageal squamous cell carcinoma (19), and gastric cancer (20). Xue et al. reported that miR-21 and miR-155 accelerated NSCLC progression through inhibiting SOCS6 expression (21). Moreover, miR-1260b directly targets SOCS6 to reduce its expression and enhance cancer cell proliferation in NSCLC $(22,23)$. However, the interaction between miR-1278 and SOCS6 and its role in NSCLC has not been established.

Our preliminary results from bioinformatic analysis suggested that both circ_0015278 and SOCS6 contain potential binding sites for miR-1278. Therefore, the potential circ_0015278/miR-1278/SOCS6 axis might be involved in the progression of NSCLC. Thus, the purpose of this study was to test the hypothesis that circ_0015278 might regulate NSCLC progression via sponging miR-1278 and thus promote the expression of SOCS6. Our study will provide potential diagnostic biomarkers and therapeutic targets for NSCLC. We present the following article in accordance with the ARRIVE reporting checklist (available at https://dx.doi.org/10.21037/atm-21-3456).

\section{Methods}

\section{Patients and specimens}

NSCLC and adjacent non-tumor tissues were collected from 62 NSCLC patients at Peking University Shenzhen Hospital and stored at $-80{ }^{\circ} \mathrm{C}$ to examine the expression of circ_0015278, miR-1278, and SOCS6. Patient survival was monitored. Written informed consent was obtained from all patients, and our study received approval from the Ethics Committee of Peking University Shenzhen Hospital. The study was conducted in accordance with the Declaration of Helsinki (as revised in 2013). Clinical pathological characteristics of NSCLC patients are shown in Table 1.

\section{Cell culture and transfection}

Human NSCLC cells including CALU3, A549, H1229 and 
Table 1 Clinical pathological characteristics of patients with nonsmall cell lung cancer

\begin{tabular}{|c|c|c|c|c|}
\hline \multirow{2}{*}{ Characteristic } & \multirow{2}{*}{$\begin{array}{l}\text { All cases } \\
\quad(n=62)\end{array}$} & \multicolumn{2}{|c|}{$\begin{array}{c}\text { Circular_0015278 } \\
\text { expression }\end{array}$} & \multirow{2}{*}{$P$ value } \\
\hline & & $\begin{array}{l}\text { Low } \\
(n=31)\end{array}$ & $\begin{array}{l}\text { High } \\
(n=31)\end{array}$ & \\
\hline \multicolumn{2}{|l|}{ Age (years) } & & & 0.445 \\
\hline$<60$ & 33 & 15 & 18 & \\
\hline$\geq 60$ & 29 & 16 & 13 & \\
\hline \multicolumn{2}{|l|}{ Gender } & & & 0.798 \\
\hline Male & 35 & 18 & 17 & \\
\hline Female & 27 & 13 & 14 & \\
\hline \multicolumn{2}{|l|}{ Tumor size $(\mathrm{cm})$} & & & 0.127 \\
\hline$<3$ & 30 & 12 & 18 & \\
\hline$\geq 3$ & 32 & 19 & 13 & \\
\hline \multicolumn{2}{|l|}{ Differentiation } & & & $0.021^{*}$ \\
\hline Well & 33 & 12 & 21 & \\
\hline Moderate/poor & 29 & 19 & 10 & \\
\hline \multicolumn{2}{|c|}{ Lymph node metastases } & & & $0.002^{*}$ \\
\hline No & 34 & 11 & 23 & \\
\hline Yes & 28 & 20 & 8 & \\
\hline \multicolumn{3}{|c|}{ Tumor, node, and metastasis stage } & & $0.039^{*}$ \\
\hline I-II & 36 & 14 & 22 & \\
\hline III-IV & 26 & 17 & 9 & \\
\hline
\end{tabular}

*, $\mathrm{P}<0.05$

H1975 and bronchial epithelial cells (HBEs) were obtained from the Cell Bank of Chinese Academy of Sciences (Shanghai, China). Cells were maintained in Dulbecco's Modified Eagle's medium from Solarbio (Beijing, China) with $10 \%$ fetal bovine serum (FBS, Solarbio, Beijing, China). For actinomycin D treatment, A549 cells were treated with actinomycin D obtained from Sigma (St. Louis, MO, USA) at $3 \mu \mathrm{g} / \mathrm{mL}$ for $0,6,12,18$, and 24 hours. Cells were collected, and RNA was extracted to assess the stability of circUBXN7. Circ_0015278 was inserted into a pLV vector, and lentiviral particles were packaged. A549 and H1229 cells with stable overexpression of circ_0015278 were generated through lentiviral transduction. In some assays, cells were transfected with miR-1278 inhibitor, siRNA against SOCS6 (si-SOCS6), or miR-1278 mimics (Ribobio, Guangzhou, China) with Lipofectamine
RNAiMAX (ThermoFisher, Waltham, MA, USA). Inhibitor nucleocapsid protein (NC), siRNA NC (si-NC), and mimics $\mathrm{NC}$ (miR-NC) were used as negative controls.

\section{A subcutaneous NSCLC xenograft mouse model}

Ten-week-old male BALB/c nude mice provided by the Animal Center of Peking University Shenzhen Hospital were used to establish a subcutaneous NSCLC xenograft mouse model as previously described with modifications (4). A549 cells were infected with circ_0015278 or empty vector lentiviral particles, and then $2 \times 10^{6}$ cells were injected subcutaneously into the left flanks. Tumor volume was monitored at days $7,14,21,28$, and 35 and calculated using the formula length $\times$ width $^{2} / 2$. Finally, mice were sacrificed, and excised tumors were weighed and cut into pieces for immunohistochemistry (IHC) staining and RNA extraction. Experiments were performed under a project license (No.: SYXK2016-0167) granted by the Ethics Committee of Peking University Shenzhen Hospital, in compliance with national or institutional guidelines for the care and use of animals. A protocol was prepared before the study without registration.

\section{RNase $^{\circledR}$ digestion}

Total RNA extracted from A549 cells was digested with $\mathrm{RNase}^{\circledR}$ from Sigma-Aldrich (St. Louis, MO, United States) at $5 \mathrm{U} / \mu \mathrm{g}$ for 20 minutes at $37^{\circ} \mathrm{C}$ for 20 minutes. Then, RNA was purified, and the quantities of circ_0015278 and KLHL20 were analyzed with real-time quantitative reverse transcription polymerase chain reaction (qRT-PCR).

\section{Cell Counting Kit-8 (CCK-8) assay}

A549 and H1229 cells with indicated transfection $\left(5 \times 10^{3}\right.$ cells) were plated and cultured in 96-well plates for 0, 24, 48 , or 72 hours. At each time point, culture medium was removed and replaced with $100 \mu \mathrm{L}$ of fresh medium. Then, CCK-8 reagents $(10 \mu \mathrm{L})$ were added into each well and incubated for an additional hours prior to recording the absorbance (450 nM). The CCK-8 kit was purchased from Dojindo (Rockville, MD, USA).

\section{5-ethynyl-2'-deoxyuridine (EdU) staining}

The Click-IT EdU kit from ThermoFisher (Waltham, MA, USA) was used for EdU staining. A549 and H1229 
cells with indicated transfection were seeded on coverslips and cultured in medium containing EdU at $10 \mu \mathrm{M}$ for 24 hours. Cells were then fixed in formaldehyde solution (4\%) and permeabilized in Triton X-100 (0.3\%). The reaction cocktail was prepared following the manual and added into cells. Cells were incubated for 30 minutes in the dark and mounted for imaging with a confocal microscope from Leica (Wetzlar, Germany).

\section{Luciferase activity}

Potential wildtype (WT) or mutant (MUT) miR-1278 binding sites in circ_0015278 and the 3' untranslated region (3' UTR) of SOCS6 were constructed into the pmirGLO vector from Promega (Madison, WI, USA). A549 and H1229 cells were co-transfected with miR-1278 mimics or miR-NC and circ_0015278 or SOCS6 luciferase reporter. After 48-72 hours, cells were harvested and used for examining the luciferase activity with the Dual-Glo system (Promega, Madison, WI, USA).

\section{Cell apoptosis analysis}

The Cell Apoptosis Detection Kit from Solarbio (Beijing, China) was used to evaluate NSCLC cell apoptosis. A549 and H1229 cells with indicated transfection $\left(1 \times 10^{5}\right.$ cells $)$ were stained with annexin V-FITC and PI in $100 \mu \mathrm{L}$ of binding buffer for 15 minutes. Subsequently, an additional $300 \mu \mathrm{L}$ of binding buffer was added, and cell apoptosis was immediately assessed with Attune NxT flow cytometer (ThermoFisher, Waltham, MA, USA).

\section{Transwell assays}

Eight $\mu \mathrm{m}$-pore chambers for transwell assays were purchased from Corning (Corning, NY, USA). The upper chamber was pre-coated with Matrigel (BD, Franklin Lakes, NJ, USA), and A549 and H1229 cells with indicated transfection $\left(2 \times 10^{5}\right.$ cells) were plated on the upper chamber and cultured for 18-24 hours for invading into the lower chamber. Then, crystal violet (Sigma, St. Louis, MO, USA) was used to stain cells in the lower chamber, and cells were imaged with an EVOS XL microscope (ThermoFisher, Waltham, MA, USA).

\section{Colony formation analysis}

A549 and H1229 cells with indicated transfection $\left(1 \times 10^{3}\right.$ cells $)$ were plated in a 6-well plate and maintained in DMEM with $10 \%$ FBS for two weeks. Medium was replaced every 2-3 days. Then, cell colonies formed by A549 and H1229 cells were washed, fixed, and stained with crystal violet (Sigma, St. Louis, MO, USA). Colonies were imaged under a BX51 microscope from Olympus (Tokyo, Japan).

\section{RNA immunoprecipitation (RIP) assay}

To perform RIP, magnetic beads were pre-coated with a rabbit Ago-2 antibody (Abcam, Cambridge, UK) or a rabbit normal IgG. A549 and H1229 cells were lysed on ice, and cell lysates were collected. Magnetic beads were added into cell lysates and incubated overnight. The Next day, RNA was eluted, and the quantities of circ_0015278 and miR1278 were evaluated with qRT-PCR.

\section{RNA pull-down}

The interactions between circ_0015278 and miR-217, miR1278, miR-142-3p, or miR-508-3p were examined using RNA pull-down assays with a magnetic RNA-Protein pulldown kit (ThermoFisher, Waltham, MA, USA). In brief, A549 and H1229 cells were lysed, and cell lysates were collected. Then, biotin-conjugated circ_0015278 probes were mixed with cell lysates and incubated with gentle rotation overnight at $4{ }^{\circ} \mathrm{C}$. The next day, streptavidinlabeled magnetic beads were added and incubated for an additional 2 hours. Finally, RNA was eluted, and the quantities of miR-217, miR-1278, miR-142-3p, and miR508-3p were determined using qRT-PCR.

\section{IHC staining}

Excised subcutaneous tumors were sliced into pieces, fixed, and embedded in paraffin. Samples were cut into $5 \mu \mathrm{m}$ sections, which were then deparaffinized and rehydrated. After antigen retrieval, sections were treated with $\mathrm{H}_{2} \mathrm{O}_{2}$ and blocked in $8 \%$ bovine serum albumin (BSA) solution. Then, sections were incubated with a rabbit Ki-67 (1:100, Abcam, Cambridge, UK) or SOCS6 (1:50, Abcam, Cambridge, UK) antibody overnight. The next day, sections were rinsed and incubated with a goat anti-rabbit horseradish peroxidase (HRP)-conjugated secondary antibody for 1 hour. Signals were visualized with diaminobenzidine (DAB, Beyotime, Shanghai, China). Finally, sections were stained with hematoxylin (Sigma, St. Louis, MO, USA), mounted, and 
Table 2 Real-time quantitative reverse transcription polymerase chain reaction primers used in our study

\begin{tabular}{ll}
\hline Primers & Primer sequences \\
\hline Circular_0015278 & 5'-AGTGATGTGGCCCCTACAAG-3' \\
& 5'-CCACTGGAGACCACTGGTTG-3' \\
MicroRNA-1278 & 5'-TAGTACTGTGCATATCATC-3' \\
& 5'-GAACATGTCTGCGTATCTC-3' \\
SOCS6 & 5'-ATCACGGAGCTATTGTCTGGA-3' \\
& 5'-CTGACTCTCATCCTCGGGGA-3' \\
U6 small nuclear RNA & 5'-CTCGCTTCGGCAGCACA-3' \\
& 5'-AACGCTTCACGAATTGCGT-3' \\
GAPDH & 5'-GGATTGGTCGTATTGGG-3' \\
& 5'-GGAAGATGGTGATGGGATT-3' \\
\hline
\end{tabular}

GAPDH, glyceraldehyde 3-phosphate dehydrogenase.

imaged under a BX51 microscope (Olympus).

\section{Western blot}

A549 and H1229 cells with indicated transfection were lysed, and cell lysates were collected followed by protein quantification using BCA Protein Assay Kit (Beyotime, Shanghai, China). Proteins $(40 \mu \mathrm{g})$ were loaded for electrophoresis for 1-2 hours and transferred to polyvinylidene difluoride (PVDF) membranes (Sigma, St. Louis, MO, USA) prior to blocking in $5 \%$ non-fat milk solution for 1 hour. Subsequently, membranes were incubated with a rabbit E-cadherin (Abcam, Cambridge, UK 1:1,000), N-cadherin (Abcam, Cambridge, UK, 1:1,000), vimentin (Abcam, Cambridge, UK, 1:2,000) or glyceraldehyde 3-phosphate dehydrogenase (GAPDH) (Abcam, Cambridge, UK, 1:5,000) antibody overnight. The next day, membranes were rinsed and incubated with an HRP-conjugated goat anti-rabbit secondary antibody (Bosterbio, Pleasanton, CA, USA) for 1 hour. Bands were visualized using enhanced chemiluminescence (ECL) substrates (Beyotime, Shanghai, China).

\section{Quantitative real-time reverse-transcription PCR (qRT- PCR)}

For RNA extraction, subcutaneous tumors from mice and patient tissues were snap-frozen and homogenized. RNA was isolated from A549 and H1229 cells with indicated transfection and tissue homogenates with TRIzol Reagent from Sigma (St. Louis, MO, USA) and quantified with Qubit 4 (ThermoFisher, Waltham, MA, USA), which was reversely transcribed into cDNA. The mirPremier microRNA Isolation Kit from Sigma (St. Louis, MO, USA) was used to extract miRNAs, and miRNAs were reversely transcribed using the miScript kit (QIAGEN, Germantown, MD, USA). The expression of circ_0015278, miR-1278, and SOSC6 was examined by quantitative real-time PCR. Circ_0015278 and SOCS were normalized to GAPDH, and miR-1278 was normalized to U6 snRNA. Primers used are listed in Table 2.

\section{Statistical analyses}

Results in our study were from at least three independent experiments and are reported as mean \pm standard deviation. The significance of variance between two groups was analyzed with the Student's $t$-test. One-way analysis of variance (ANOVA) was used for comparing the variance of multiple groups. The Kaplan-Meier plotter was applied to assess the survival of patients with high or low level of circ_0015278. Spearman's correlations were used to analyze the correlations among the expression of circ_0015278, miR-1278, and SOCS6 in NSCLC tissues from patients. $\mathrm{P}<0.05$ was considered to indicate statistical significance $\left({ }^{*} \mathrm{P}<0.05,{ }^{* *} \mathrm{P}<0.01\right.$ and $\left.{ }^{* * *} \mathrm{P}<0.001\right)$.

\section{Results}

\section{The expression of circ_0015278 was reduced in NSCLC tissues and cells}

To explore the function of circular RNAs (circRNAs) in NSCLC, we reanalyzed circRNA expression profiles in NSCLC and adjacent normal tissues from three microarrays, GSE112214, GSE158659, and GSE101586, and found six downregulated circRNAs in NSCLC tissues (Figure 1A). We collected NSCLC and adjacent nontumor tissues from patients and analyzed the expression of circ_0015278 by qRT-PCR. Circ_0015278 was significantly downregulated in NSCLC tissues (Figure 1B). NSCLC patients were divided into two groups based on the median of circ_0015278 expression in NSCLC tissues: circ_0015278 $8^{\text {high }}$ and circ_0015278 $8^{\text {low }}$. We observed obvious poor survival of circ_0015278 $8^{\text {low }}$ patients (Figure 1C), indicating that circ_0015278 improved the prognosis of patients with NSCLC. Furthermore, we found that circ_0015278 expression was closely correlated with tumor size, lymphatic 
A
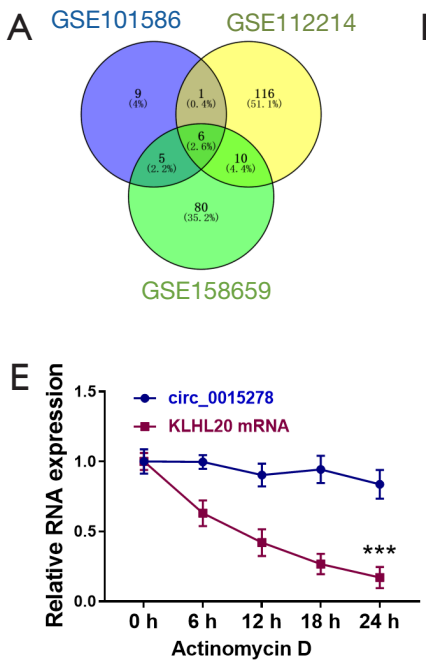
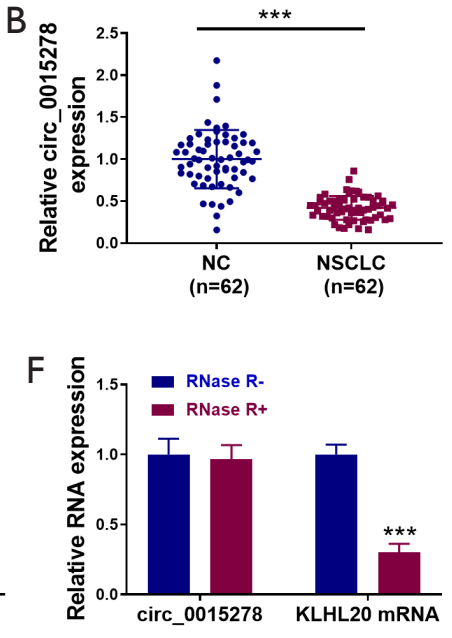
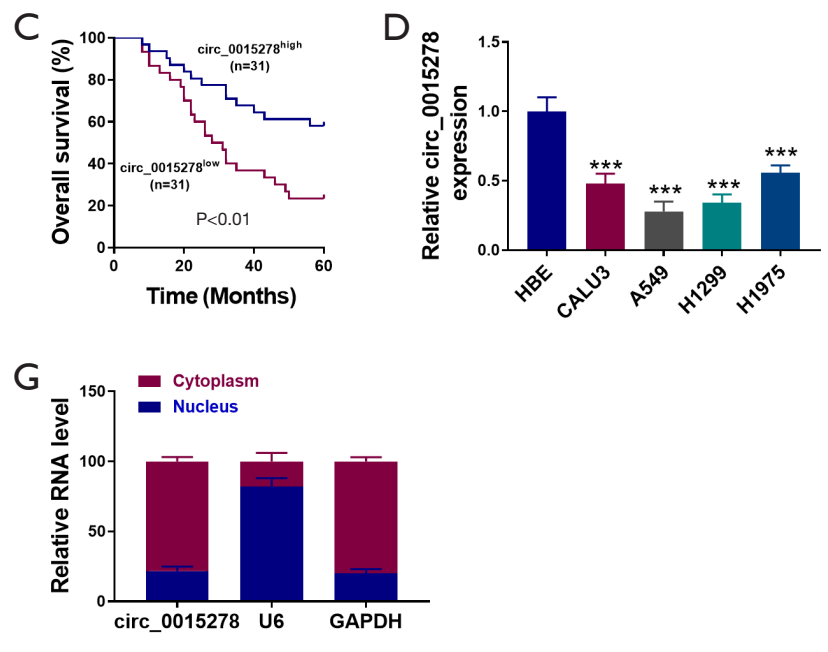

Figure 1 Circular (circ)_0015278 was downregulated in non-small cell lung cancer (NSCLC) tissues and cells. (A) Downregulated circular RNAs in GSE112214, GSE158659, and GSE101586 were analyzed. (B) Circ_0015278 expression in NSCLC and adjacent normal tissues was assessed by real-time quantitative reverse transcription polymerase chain reaction (qRT-PCR) (n=62). (C) A Kaplan-Meier plotter was used to evaluate the survival of patients with high and low expression of circ_0015278 (circ_0015278 ${ }^{\text {high }}$ and circ_0015278 ${ }^{\text {low }}$ ). (D) Circ_0015278 expression in CALU3, A549, H1229, H1975, and HBEs was examined with qRT-qPCR (n=3). (E) Circ_0015278 and KLHL20 messenger RNA were examined after actinomycin D treatment at 0, 6, 8, 12, 18, and 24 hours (n=3). (F) qRT-PCR analysis of circ_0015278 and KLHL20 after RNase ${ }^{\circledast}$ or mock digestion (n=3). (G) qRT-PCR analysis of circ_0015278, GAPDH, and U6 snRNA in nuclear and cytoplasmic fractions from A549 cells $(\mathrm{n}=3)$. ${ }^{* * *} \mathrm{P}<0.001$.

metastasis, and tumor, node, and metastasis (TNM) staging rather than age, gender, and tumor differentiation (Table 1). We also examined the expression of circ_0015278 in human NSCLC cell lines including CALU3, A549, H1299, and H1975 and HBEs and found that the expression of circ_0015278 was reduced in all these NSCLC cell lines compared to those of HBEs (Figure 1D). A549 and H1229 cells showed lowest expression of circ_0015278 (Figure 1D), and these were chosen for subsequent experiments. Circ_0015278 was stable in response to actinomycin D treatment and its half-life was over 24 hours, but the halflife of linear KLHL20 messenger RNA (mRNA) was less than 12 hours (Figure 1E). Furthermore, as $\mathrm{RNase}^{\circledR}$ can digest linear RNAs but not circRNAs, total RNA extracted from A549 cells were treated with $\mathrm{RNase}^{\circledR}$ and analyzed by qRT-PCR. As shown in Figure 1F, circ_0015278 was resistant to RNase ${ }^{\circledR}$ treatment, but KLHL20 mRNA was degraded, implying that circ_0015278 was a circular RNA. Nuclear and cytoplasmic fractions of A549 cells were separated, and we found that circ_0015278 was mainly located in the cytoplasm as the cytoplastic reference GAPDH (Figure 1G). The nuclear reference U6 was located in the nucleus as expected (Figure 1G). These data suggested that circ_0015278 is a circular RNA, and that it might be implicated in the progression of NSCLC.

\section{Overexpression of circ_0015278 restrained NSCLC cell proliferation, invasion, and EMT and induced cell apoptosis}

Circ_0015278 was stably overexpressed through lentiviral transduction in NSCLC cells (Figure 2A). CCK-8 and EdU staining assays showed that overexpression of circ_0015728 markedly suppressed A549 and H1229 cell proliferation (Figure 2B,2C). Moreover, the colony formation of A549 and H1229 cells was inhibited by overexpression of circ_0015728 (Figure 2D). A549 and H1229 cells with circ_0015728 overexpression exhibited increased cell apoptosis compared to cells transfected with vectors (Figure $2 E$ ), indicating that circ_0015728 enhanced NSCLC cell apoptosis. Transwell assays with Matrigel were conducted to evaluate cell invasion. The invasive capacity of A549 and H1229 cells was restrained by overexpression of circ_0015728 (Figure 2F). Besides, we examined EMT markers such as E-cadherin, $\mathrm{N}$-cadherin, and vimentin and found that overexpression of circ_0015728 reduced 

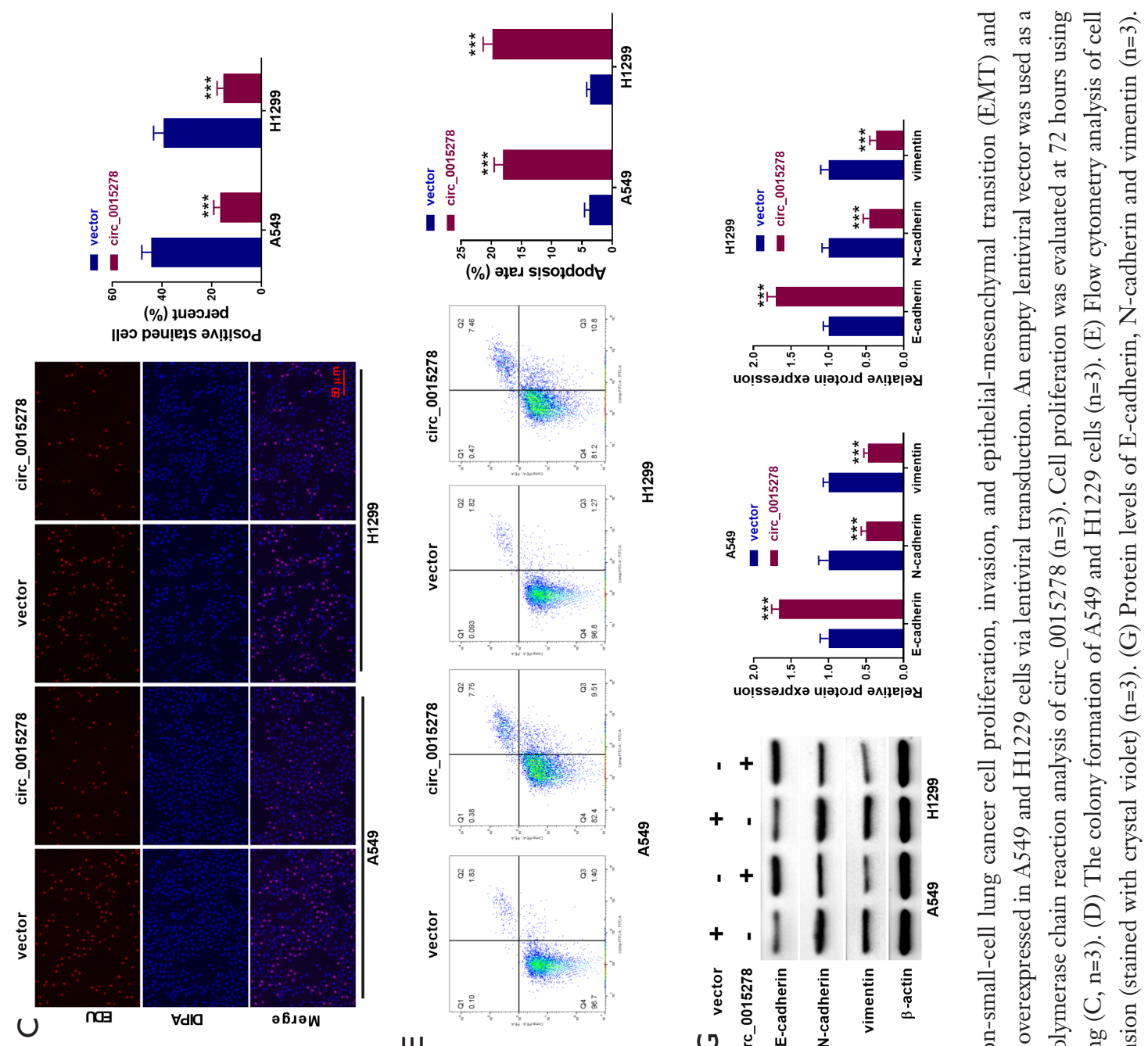

$\infty$

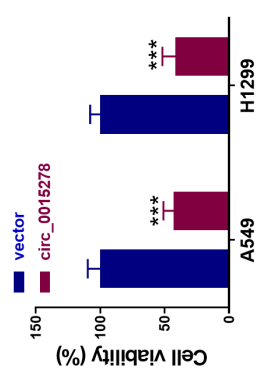

ш
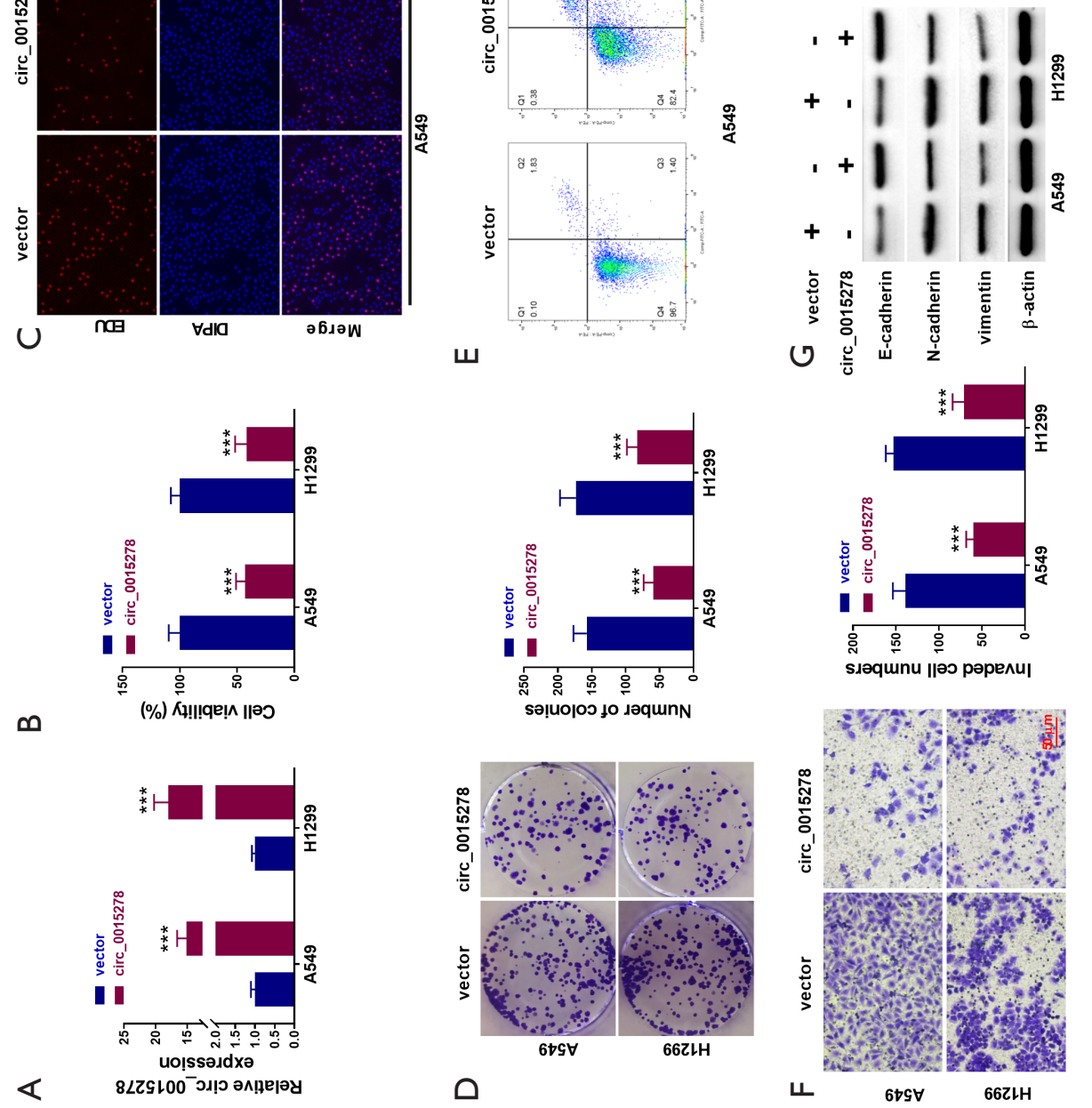

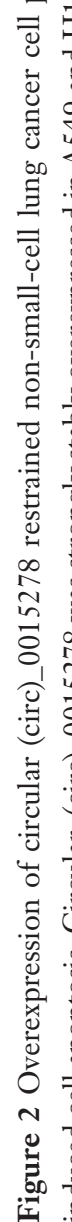

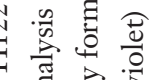

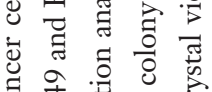

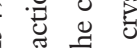

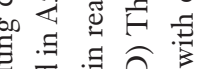
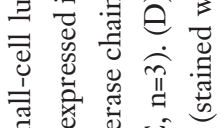

要证

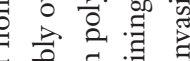

䜦

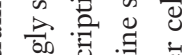

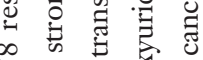

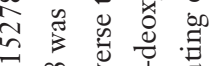

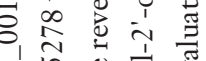

în.

至

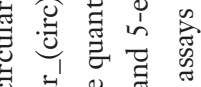

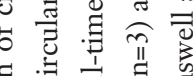

워

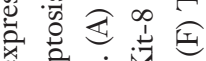

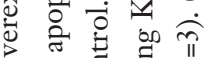

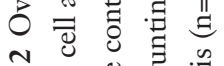

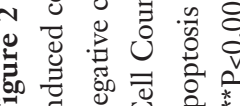


the expression of $\mathrm{N}$-cadherin and vimentin but promoted E-cadherin expression in A549 and H1229 cells (Figure 2G). These observations indicated that overexpression of circ_0015278 inhibited NSCLC cell proliferation, invasion, and EMT and enhanced cancer cell apoptosis.

\section{Circ_0015728 functioned as a miR-1278 sponge to reduce its expression in NSCLC cells}

As circRNA can function as a ceRNA to sponge miRNAs in various cancers (4), we performed bioinformatic analysis of data from circBank, starBase and circinteractome and identified four potential miRNA targets of circ_0015228 through Venn diagrams: miR-217, miR-1278, miR-142$3 \mathrm{p}$, and miR-508-3p (Figure 3A). Compared to a control probe, the circ_0015228 probe efficiently enriched miR1278, but not miR-217, miR-142-3p, and miR-508-3p, in A549 and H1229 cells (Figure 3B). In addition, miR1278 was highly expressed in NSCLC tissues (Figure 3C), and the expression of circ_0015228 negatively correlated with miR-1278 expression in NSCLC tissues (Figure 3D). Compared to HBEs, all NSCLC cells showed elevated expression of miR-1278 expression (Figure 3E). MiR-1278 was overexpressed in A549 and H1229 cells by miR-1278 mimics transfection (Figure 3F). As shown in Figure 3G, a potential binding site for miR-1278 in circ_0015228 was predicted. The binding site was mutated for constructing mutant circ_0015228 reporter. Overexpression of miR-1278 significantly reduced the luciferase activity of the wildtype circ_0015228 reporter, but the luciferase activity of the mutant circ_0015228 reporter was unaffected (Figure 3H). Furthermore, RIP assays showed that both miR-1278 and circ_0015228 could be enriched by an Ago-2 antibody (Figure 3I). The expression of miR-1278 was clearly suppressed by overexpression of circ_0015228 in A549 and H1229 cells (Figure 37). Collectively, circ_0015228 sponged miR-1278 to inhibit its expression in NSCLC cells.

\section{Overexpression of miR-1278 reversed circ_0015728- mediated suppression of NSCLC}

To examine whether circ_0015728-mediated suppression of NSCLC is dependent on sponging miR-1278, A549, and H1229 cells were transfected with circ_0015728 in combination with miR-1278 mimics or mimics NC. Circ_0015278-mediated suppression of miR-1278 expression was restored by miR-1278 mimics (Figure 4A). CCK-8 and EdU staining assays showed that circ_0015728 mediated suppressive effects on A549 and H1229, and that cell proliferation was largely reversed by miR-1278 overexpression (Figure 4B,C). Consistent with this, overexpression of circ_0015728 impaired the colony forming capacity of A549 and H1229 cells, but it was reversed by concomitant overexpression of miR-1278 (Figure 4D). Increased apoptosis of A549 and H1229 cells with circ_0015728 overexpression was partially suppressed by miR-1278 overexpression (Figure 4E). Moreover, overexpression of miR-1278 reversed circ_0015728-mediated inhibition of the invasion of A549 and H1229 cells (Figure 4F). Decreased expression of $\mathrm{N}$-cadherin and vimentin and elevated E-cadherin expression were observed in A549 and H1229 cells with circ_0015728 overexpression, which was restored by overexpression of miR1278 (Figure 4G). To conclude, circ_0015728 attenuated NSCLC cell proliferation, invasion, and EMT and enhanced its apoptosis through sponging miR-1278.

\section{MiR-1278 targeted SOCS6 to suppress its expression in NSCLC cells}

Through TargetScan, we predicted a potential binding site for miR-1278 in the 3' UTR of SOCS6 (Figure 5A) and that overexpression of miR-1278 could reduce the luciferase activity of the wildtype SOCS6 reporter, but it did not affect the luciferase activity of the mutant SOCS6 reporter in A549 and H1229 cells (Figure 5B). Besides, the expression of SOCS6 was clearly reduced in A549 and H1229 cells with miR-1278 overexpression (Figure 5C). Overexpression of circ_0015728 promoted SOCS6 expression in A549 and H1229 cells, which was inhibited by concomitant miR1278 overexpression (Figure 5D). Compared to adjacent normal tissues, NSCLC tissues showed reduced expression of SOCS6 (Figure 5E). Furthermore, we found that the expression of SOCS6 negatively correlated with miR-1278 expression but positively correlated with circ_0015728 expression in NSCLC tissues from patients (Figure $5 F, G$ ). We also analyzed SOCS6 expression in NSCLC cells and HBEs and found that SOCS6 was downregulated in NSCLC cells comparted to that in HBEs (Figure 5H). These observations suggest that miR-1278 could bind to SOCS6 to inhibit its expression in NSCLC cells and circ_0015728 might regulate SOCS6 expression through targeting miR-1278.

\section{Knockdown of SOCS6 reversed miR-1278 inbibitor- mediated acceleration of NSCLC}

To investigate whether miR-1278 regulates NSCLC 

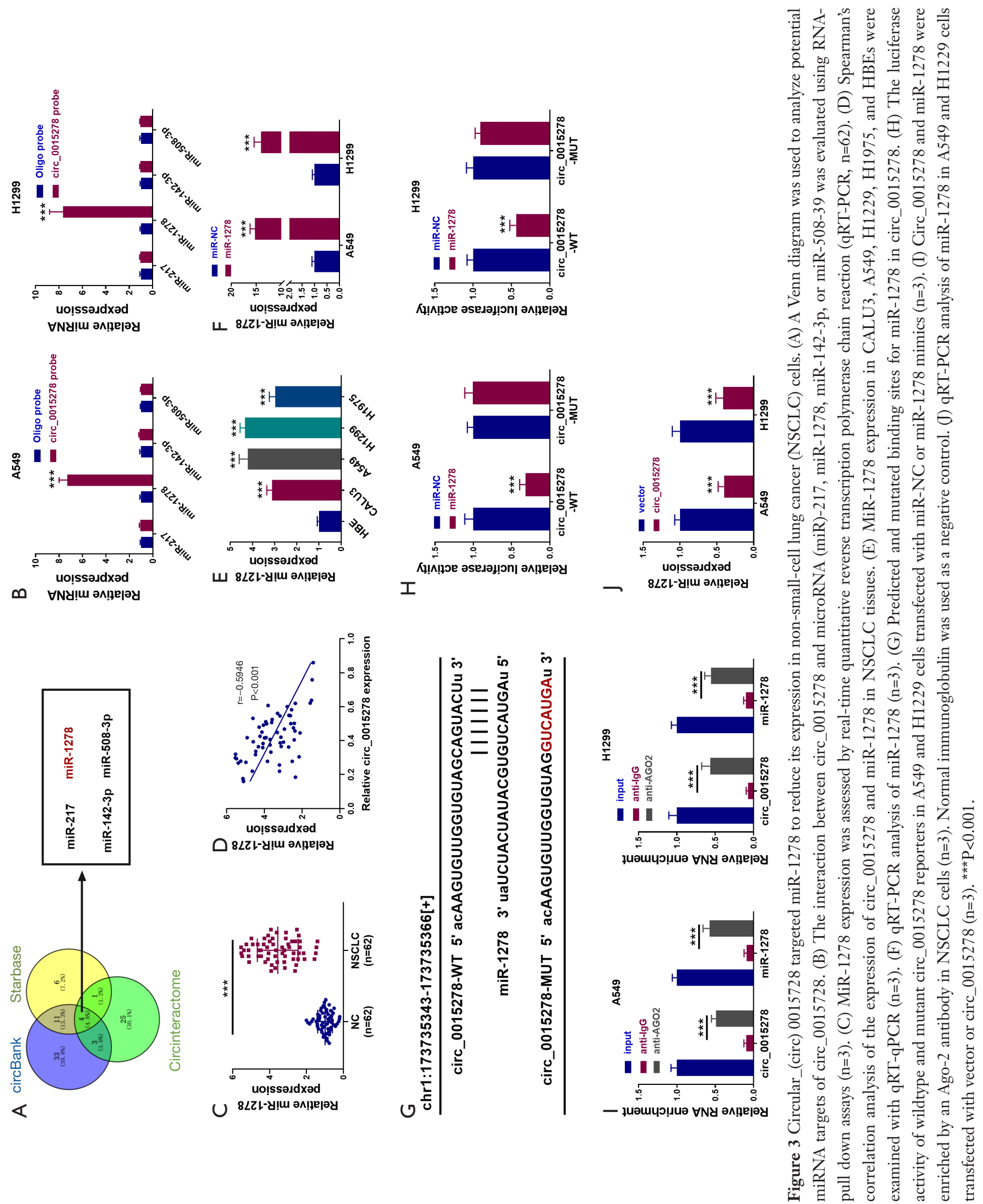

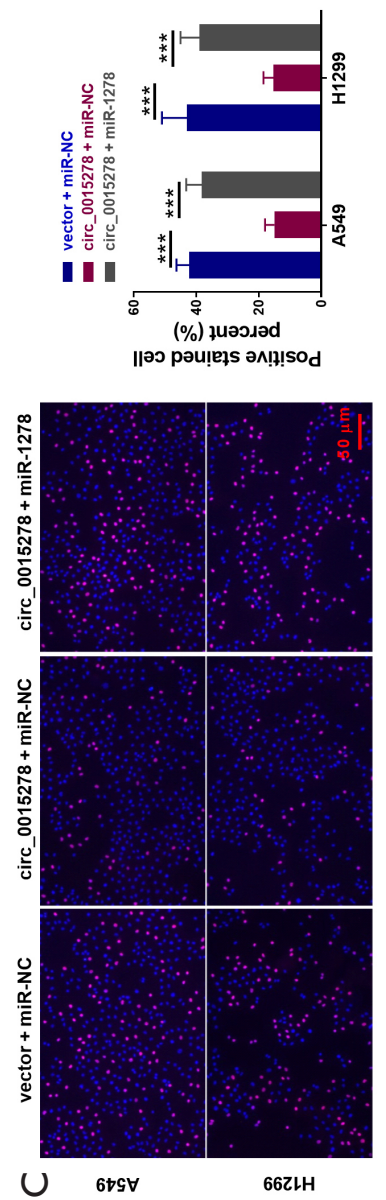

$\infty$
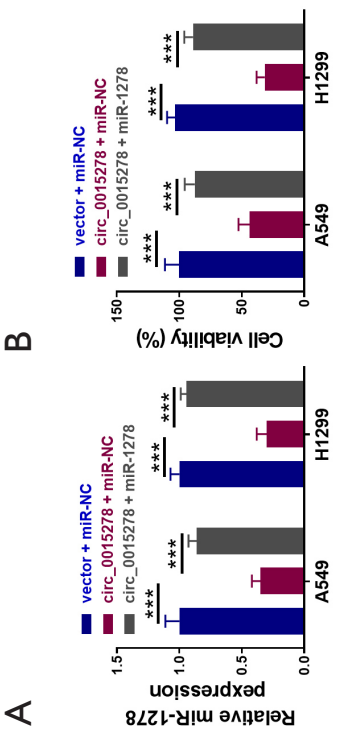
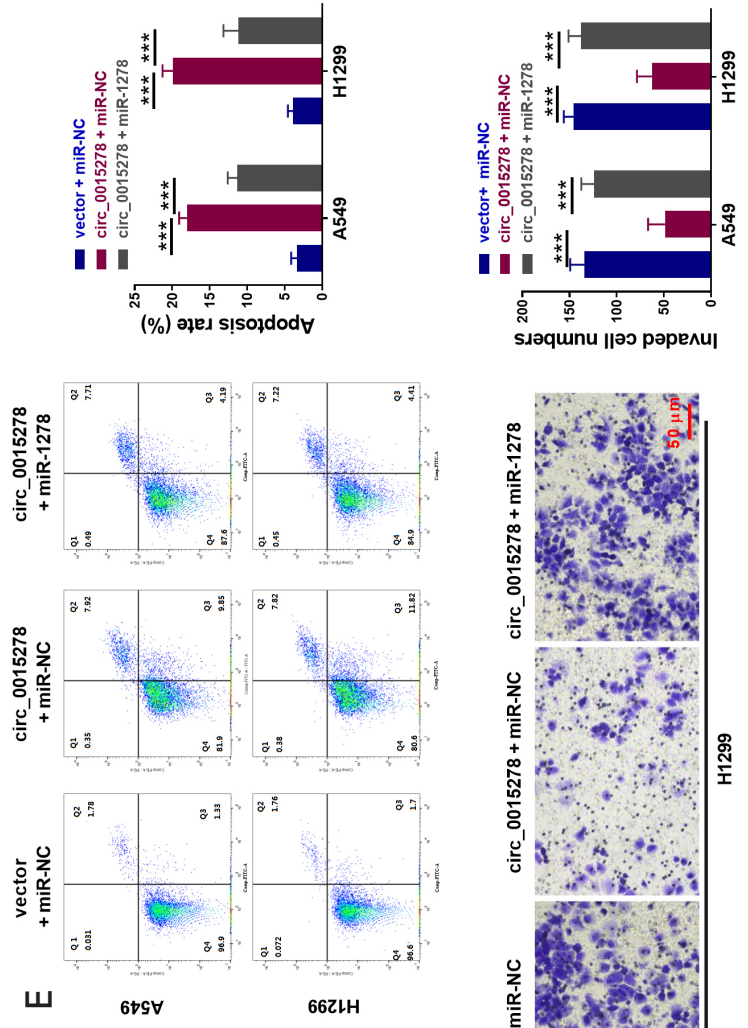

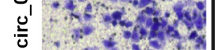

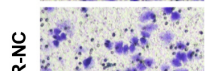

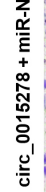
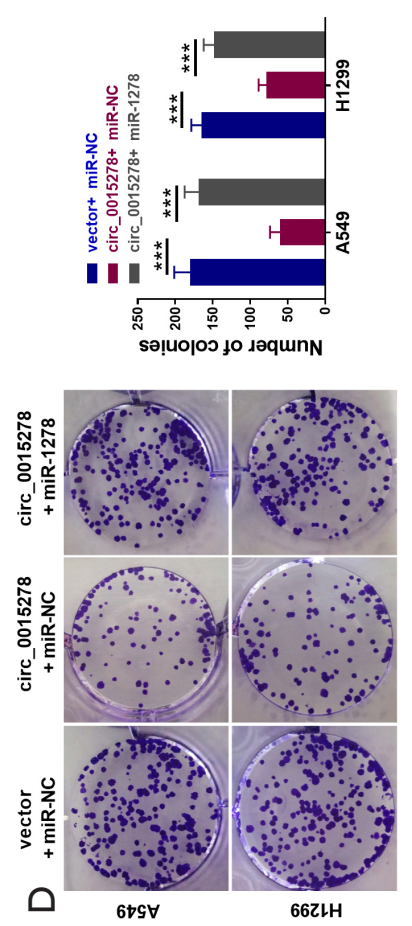

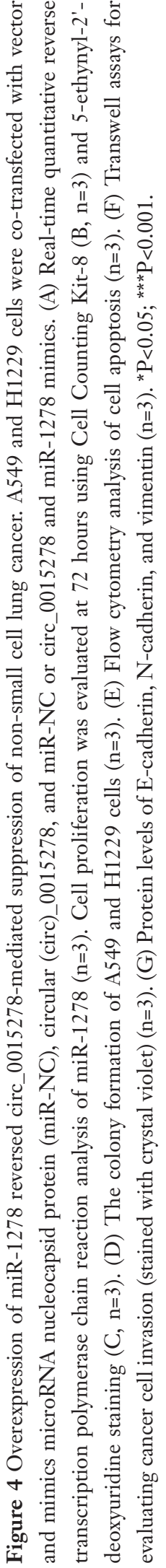



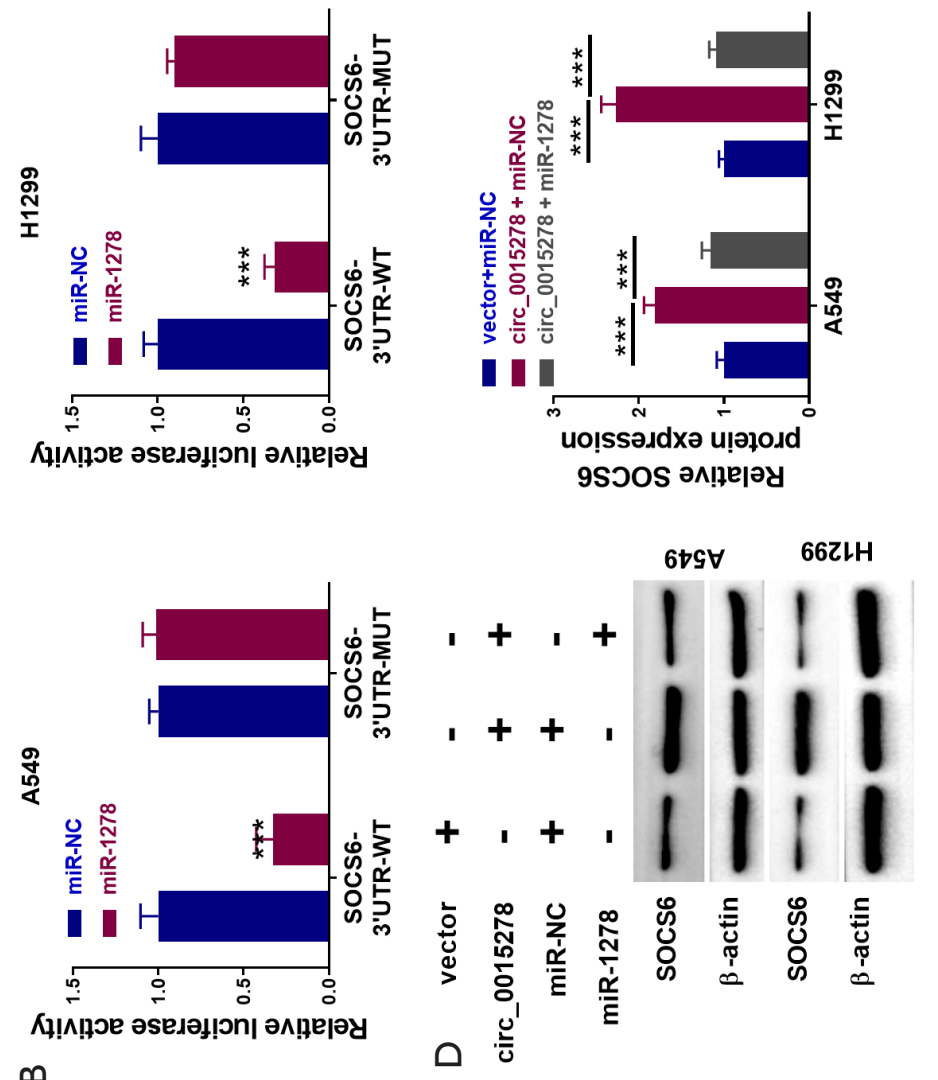

$\infty$

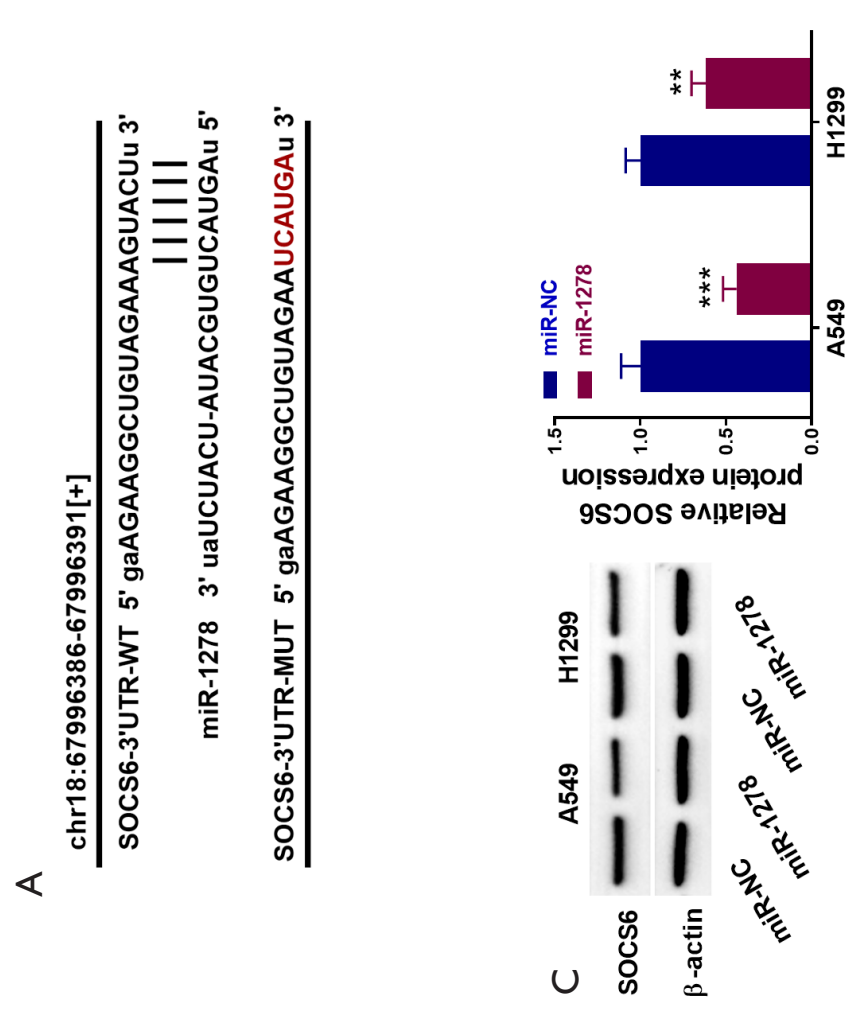

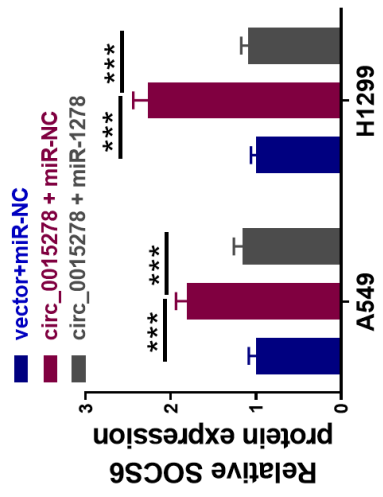
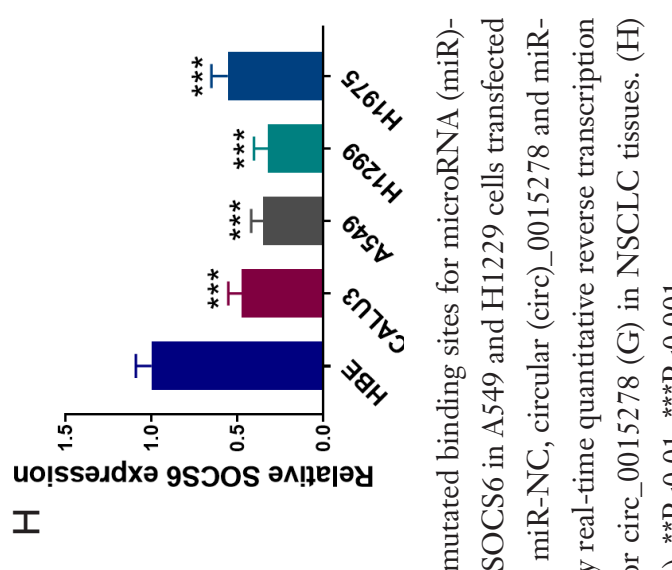

$\exists$ is

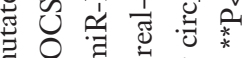

घ

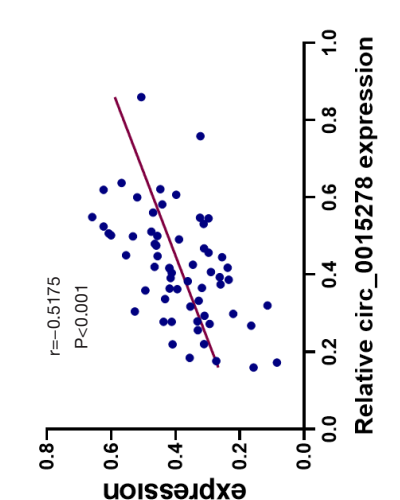

৩ 9soos әм!ฺерәу

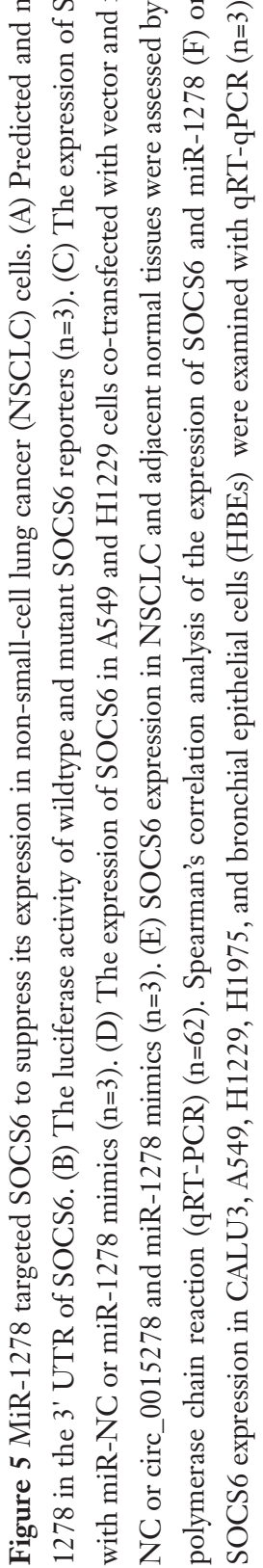


development by inhibiting SOCS6 expression, SOCS6 was knocked down through si-SOCS6 transfection (Figure 6A). Subsequently, NSCLC cells were cotransfected with miR-1278 inhibitor and si-SOCS6 or siNC. Knockdown of miR-1278 inhibited proliferation and colony formation, which were all reversed via concomitant silencing of SOCS6 (Figure 6B,6C). We observed enhanced apoptosis of A549 and H1229 cells transfected with miR1278 inhibitor, but it was partially reduced by si-SOCS6 transfection (Figure 6D,6E). Additionally, knockdown of miR-1278 inhibited the invasion of NSCLC cells, which was largely reversed by knockdown of SOCS6 (Figure $6 F$ ). Reduced expression of $\mathrm{N}$-cadherin and vimentin and increased E-cadherin expression in A549 and H1229 cells with knockdown of miR-1278 were restored by concomitant silencing of SOCS6 (Figure 6G). These data indicate that miR-1278 regulated the progression of NSCLC by inhibiting SOCS6 expression.

\section{Circ_0015728 inbibited the growth of NSCLC in vivo}

A subcutaneous NSCLC xenograft mouse model was established to examine whether circ_0015728 could regulate the progression of NSCLC in vivo. Circ_0015278 was stably overexpressed through lentiviral transduction in A549 cells, and cells were injected subcutaneously into the left flanks of mice. The volume and weight of subcutaneous tumors formed by A549 cells with circ_0015278 overexpression were significantly reduced compared to those of tumors formed by cells transfected with vector (Figure $7 A, 7 B)$. IHC staining showed reduced expression of $\mathrm{Ki}-67$ in tumors formed by A549 cells with circ_0015278 overexpression, indicating that circ_0015278 suppressed tumor cell proliferation (Figure 7C). Moreover, circ_0015278 overexpressing tumors showed increased expression of SOCS6 (Figure 7C). As expected, circ_0015278 was upregulated in tumors formed by A549 cells transfected with circ_0015278 overexpressing lentiviral particles (Figure 7D). In addition, miR-1278 expression was reduced in circ_0015278 overexpressing tumors, suggesting that circ_0015278 suppressed miR-1278 expression in tumors. Taken together, circ_0015278 restrained the growth of NSCLC possibly through targeting the miR-1278/SOCS6 axis in vivo.

\section{Discussion}

Lung carcinoma is common and fatal worldwide (24). In
2018 more than 2 million new patients were diagnosed, and lung carcinoma caused over 1.7 million deaths (25). As the most common type of lung carcinoma, NSCLC is highly lethal, as most patients have advanced cancer when they are diagnosed (26). Thus, it is truly important to explore the pathogenesis of NSCLC and identify key regulators of NSCLC progression for seeking novel diagnostic biomarkers and therapeutic targets. In the present study, we showed key roles of the novel circ_0015278/miR-1278/SOCS6 axis in regulating the progression of NSCLC for the first time. We showed that circ_0015278 was downregulated, and its overexpression suppressed malignant phenotypes of NSCLC by sponging miR-1278. Furthermore, miR-1278 regulated the progression of NSCLC by binding to SOCS6 and reducing its expression. To conclude, circ_0015278 was found to exert anti-tumor effects in NSCLC via regulating the miR-1278/SOCS6 axis.

CircRNA is becoming a research focus as increasing evidence in recent decades has shown the key roles of circRNA in many aspects of tumor biology (27). Emerging studies increasingly show the potential of circRNA to be applied in personalized cancer treatment $(28,29)$. Many circRNAs are implicated in the regulation of the progression of NSCLC (30). For instance, circSLC25A16 promotes the glycolysis and proliferation of NSCLC cells via interacting with miR-488-3p and enhancing the expression of HIF-1 $\alpha$ (31). CircPTPRA as been reported to inhibit EMT and metastasis through targeting miR-96-5p and thus increasing RASSF8 expression in NSCLC cells (32). In addition, circRNAs in plasma and tumor tissues are considered to have diagnostic and prognostic value for NSCLC, such as circFARSA (33), circ_0014130 (34), and circ_100876 (35). To seek novel circRNA candidates with diagnostic and therapeutic values for NSCLC, we considered circ_0015278 due to its low expression in NSCLC tissues. Intriguingly, consistent with previous findings that show that circ_0015278 functions as a tumor suppressor in NSCLC (11), we demonstrated that circ_0015278 overexpression restrained the progression of NSCLC both in vitro and in vivo, indicating diagnostic and therapeutic value of circ_0015278 in relation to NSCLC.

CircRNAs can sponge miRNAs and regulate downstream targets in cancers. Several miRNA targets of circ_0015278 have been identified, such as miR-1228, miR-141-3p, and miR-200a-3p (36). Here, we identified miR-1278 as a novel miRNA target of circ_0015278 in NSCLC. Circular 0015278-mediated suppression of NSCLC progression was dependent on targeting miR-1278 (37). MiR-1278 was 

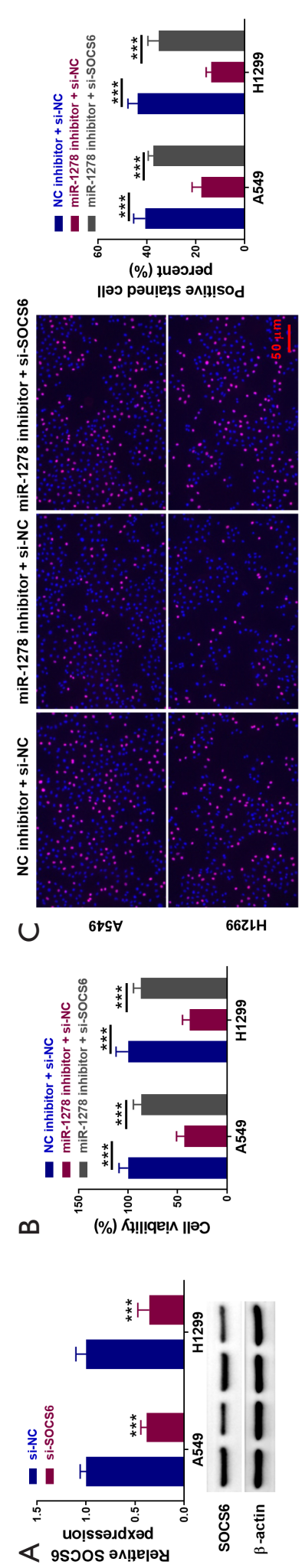
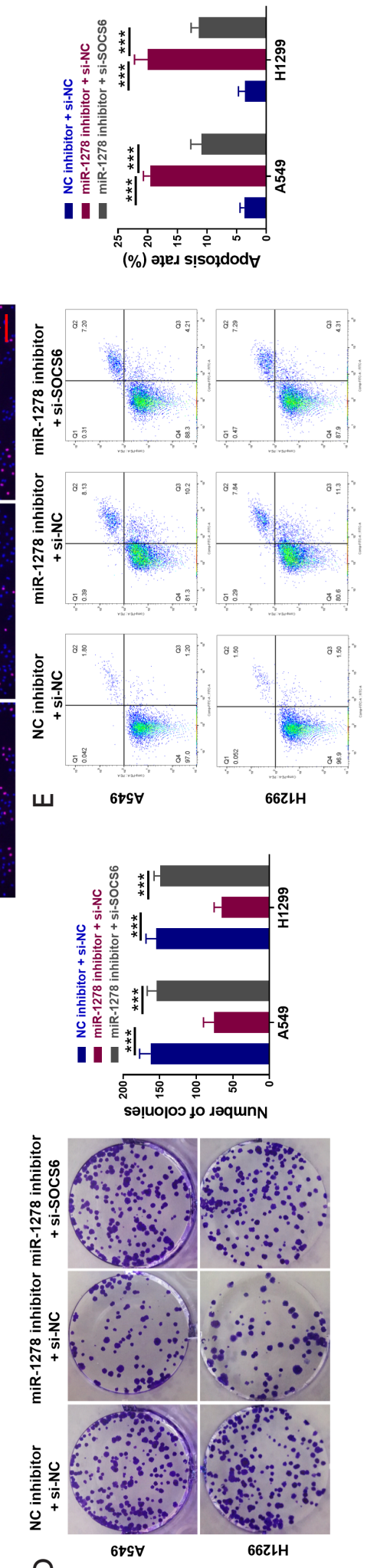
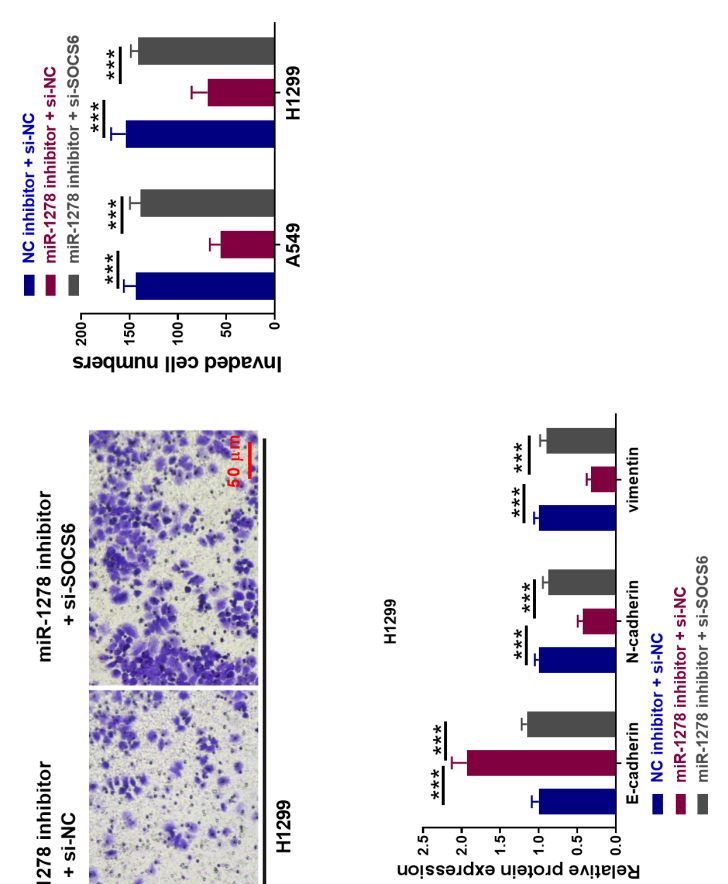

$\Xi \infty$

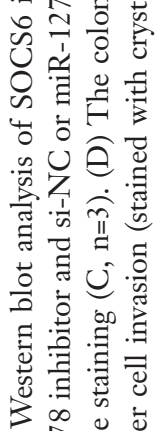

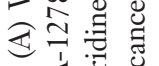

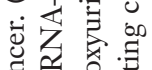
픙 웡 so 论室

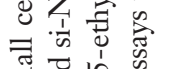

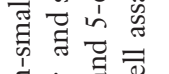

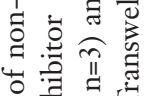
을

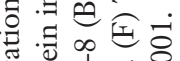
宸

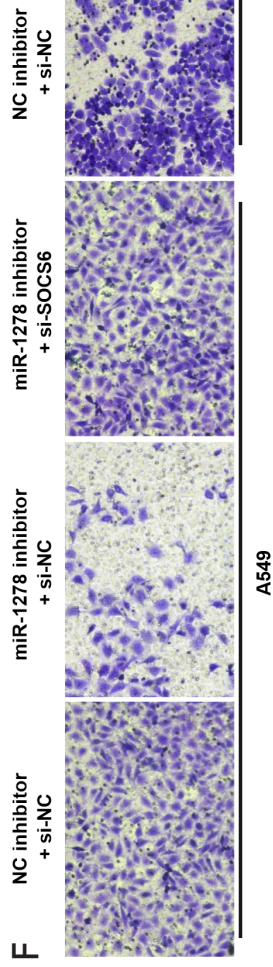

政

尊运语守

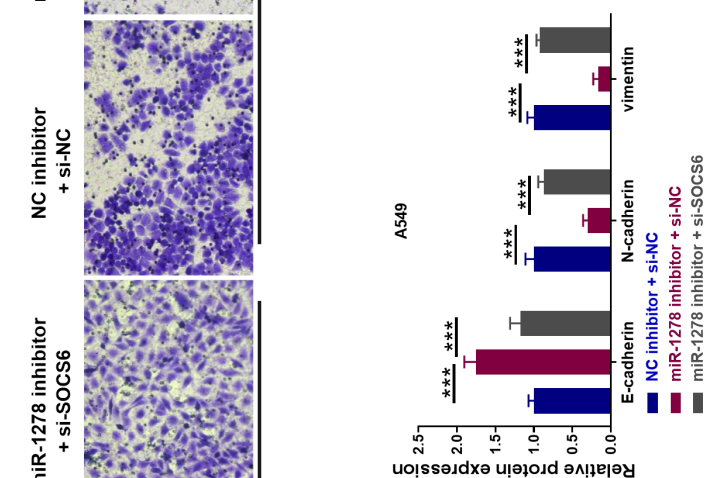

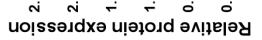

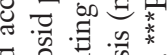

월

券守

윰.

च 0

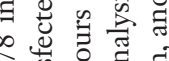
ป 政

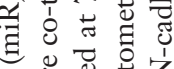
造范范

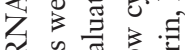

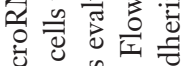
苗嵒画

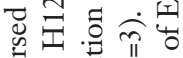
西造

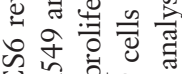
눙 कิึ पै $\triangleq 0^{\circ}$ 토월 范 O

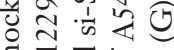

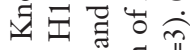

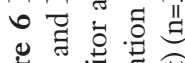

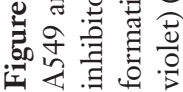

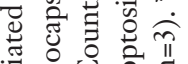



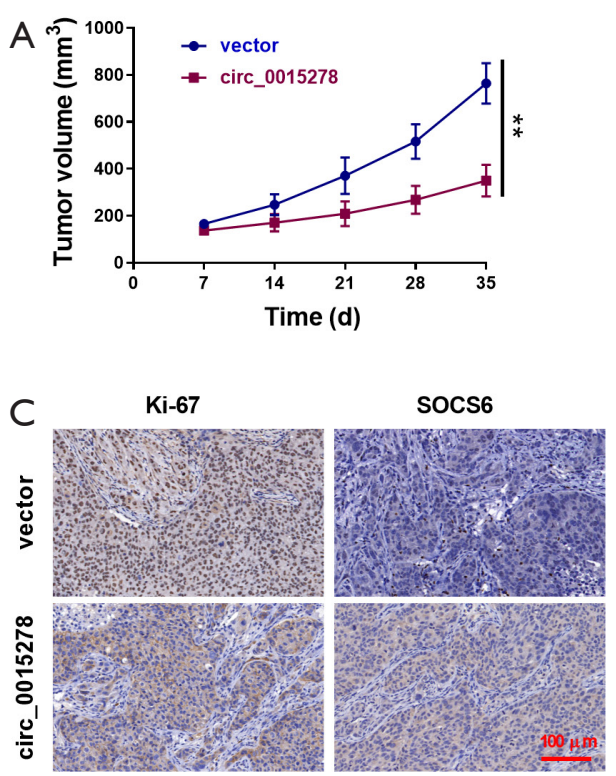
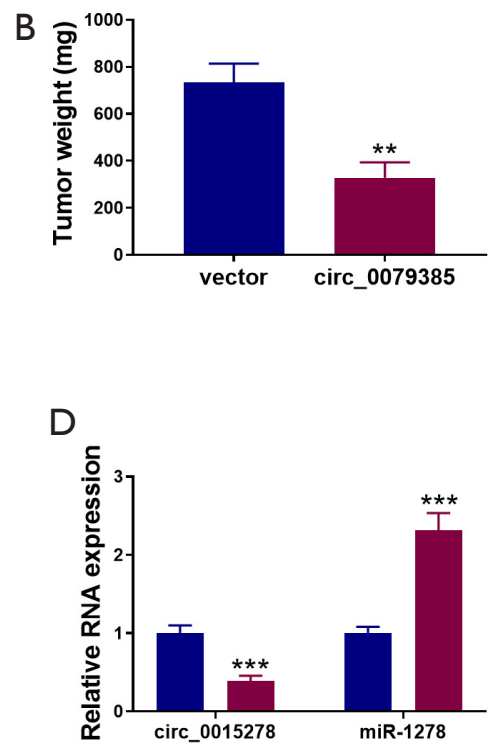

Figure 7 Circular (circ)_0015728 inhibited the growth of non-small cell lung cancer in vivo. Circ_0015278 was stably overexpressed in A549 cells via lentiviral transduction. An empty lentiviral vector was used as a negative control. Then cells were injected subcutaneously into the left flanks. Tumor volume $(A, n=8)$ and weight $(B, n=8)$. (C) Immunohistochemistry staining of Ki-67 and SOCS6 in tumor sections. (D) Real-time quantitative reverse transcription polymerase chain reaction analysis of circ_0015278 and microRNA-1278 in subcutaneous tumors $(\mathrm{n}=3) .{ }^{* *} \mathrm{P}<0.01 ;{ }^{* * *} \mathrm{P}<0.001$.

reported to exert anti-tumor activity in nasopharyngeal and colorectal cancers, but we are the first to report that miR-1278 had an opposing oncogenic role in NSCLC. Generally speaking, a single miRNA is able to target many downstream mRNAs and the opposing activity of miR1278 might be caused by different mRNA targets in various contexts of diverse cancers (38).

SOCS6 has been well documented as a tumor suppressor in carcinomas including NSCLC (39). Consistent with this, we reported SOCS6 as a novel target of miR-1278, and SOCS6 silencing partially reversed knockdown of miR-1278mediated suppression of NSCLC malignant phenotypes, demonstrating the anti-tumor activity of SOCS6 in NSCLC. It also explains why miR-1278 exerted an oncogenic role in NSCLC. SOCS6 can regulate several signaling pathways, such as KIT, Flt3, and STAT3 signaling (40). Further studies are needed to investigate downstream signaling of SOCS6 in NSCLC for clinical application.

To summarize, we are the first to demonstrate that circ_0015278 suppressed the proliferation, invasion, and EMT of NSCLC and enhanced NSCLC cell apoptosis through sponging miR-1278 and thus increased the expression of SOCS6. Our findings not only shed light on the molecular basis of NSCLC progression but also provide potential diagnostic biomarkers and therapeutic targets. In the future, we can develop small molecule compounds for targeted therapy of circ_0015278 and miR-1278, or develop corresponding detection kits for the above small molecules in clinical applications. However, more investigations are required to clarify molecular mechanisms for future clinical applications.

\section{Acknowledgments}

Funding: None.

\section{Footnote}

Reporting Checklist: The authors have completed the ARRIVE reporting checklist. Available at https://dx.doi. org/10.21037/atm-21-3456

Data Sharing Statement: Available at https://dx.doi. org/10.21037/atm-21-3456

Conflicts of Interest: All authors have completed the ICMJE uniform disclosure form (available at https://dx.doi. org/10.21037/atm-21-3456). The authors have no conflicts 
of interest to declare.

Ethical Statement: The authors are accountable for all aspects of the work in ensuring that questions related to the accuracy or integrity of any part of the work are appropriately investigated and resolved. Written informed consent was obtained from all patients, and our study received approval from the Ethics Committee of Peking University Shenzhen Hospital. The study was conducted in accordance with the Declaration of Helsinki (as revised in 2013). Experiments were performed under a project license (No.: SYXK2016-0167) in compliance with national and institutional guidelines for the care and use of animals.

Open Access Statement: This is an Open Access article distributed in accordance with the Creative Commons Attribution-NonCommercial-NoDerivs 4.0 International License (CC BY-NC-ND 4.0), which permits the noncommercial replication and distribution of the article with the strict proviso that no changes or edits are made and the original work is properly cited (including links to both the formal publication through the relevant DOI and the license). See: https://creativecommons.org/licenses/by-nc-nd/4.0/.

\section{References}

1. Latimer KM, Mott TF. Lung cancer: diagnosis, treatment principles, and screening. Am Fam Physician 2015;91:250-6.

2. Cersosimo RJ. Lung cancer: a review. Am J Health Syst Pharm 2002;59:611-42.

3. Molina JR, Yang P, Cassivi SD, et al. Non-small cell lung cancer: epidemiology, risk factors, treatment, and survivorship. Mayo Clin Proc 2008;83:584-94.

4. Miller KD, Nogueira L, Mariotto AB, et al. Cancer treatment and survivorship statistics, 2019. CA Cancer J Clin 2019;69:363-85.

5. Zappa C, Mousa SA. Non-small cell lung cancer: current treatment and future advances. Transl Lung Cancer Res 2016;5:288-300.

6. Kristensen LS, Andersen MS, Stagsted LVW, et al. The biogenesis, biology and characterization of circular RNAs. Nat Rev Genet 2019;20:675-91.

7. Ren G, Zhao Q, Yan C, et al. Circular RNA circZFR promotes tumorigenic capacity of lung cancer via CCND1. Transl Cancer Res 2020;9:3303-11.

8. Zhang C, Ma L, Niu Y, et al. Circular RNA in Lung Cancer Research: Biogenesis, Functions, and Roles. Int J
Biol Sci 2020;16:803-14.

9. Jiang MM, Mai ZT, Wan SZ, et al. Microarray profiles reveal that circular RNA hsa_circ_0007385 functions as an oncogene in non-small cell lung cancer tumorigenesis. J Cancer Res Clin Oncol 2018;144:667-74.

10. Zhang Y, Zhao H, Zhang L. Identification of the tumorsuppressive function of circular RNA FOXO3 in nonsmall cell lung cancer through sponging miR155. Mol Med Rep 2018;17:7692-700.

11. Li L, Sun D, Li X, et al. Identification of Key circRNAs in Non-Small Cell Lung Cancer. Am J Med Sci 2021;361:98-105.

12. Chen D, Ma W, Ke Z, et al. CircRNA hsa_circ_100395 regulates miR-1228/TCF21 pathway to inhibit lung cancer progression. Cell Cycle 2018;17:2080-90.

13. Bach DH, Lee SK, Sood AK. Circular RNAs in Cancer. Mol Ther Nucleic Acids 2019;16:118-29.

14. Sun X, Wang M, Xu R, et al. Prognostic model based on circular RNA circPDK1 for resected lung squamous cell carcinoma. Transl Lung Cancer Res 2019;8:907-19.

15. Li X, Lin S, Mo Z, et al. CircRNA_100395 inhibits cell proliferation and metastasis in ovarian cancer via regulating miR-1228/p53/epithelial-mesenchymal transition (EMT) axis. J Cancer 2020;11:599-609.

16. Cheng X, Qiu J, Wang S, et al. Comprehensive circular RNA profiling identifies CircFAM120A as a new biomarker of hypoxic lung adenocarcinoma. Ann Transl Med 2019;7:442.

17. Zhao Y, Wang P, Wu Q. miR-1278 sensitizes nasopharyngeal carcinoma cells to cisplatin and suppresses autophagy via targeting ATG2B. Mol Cell Probes 2020;53:101597.

18. Lin W, Zou H, Mo J, et al. Micro1278 Leads to Tumor Growth Arrest, Enhanced Sensitivity to Oxaliplatin and Vitamin D and Inhibits Metastasis via KIF5B, CYP24A1, and BTG2, Respectively. Front Oncol 2021;11:637878.

19. Yuan D, Wang W, Su J, et al. SOCS6 Functions as a Tumor Suppressor by Inducing Apoptosis and Inhibiting Angiogenesis in Human Prostate Cancer. Curr Cancer Drug Targets 2018;18:894-904.

20. Sun X, Sun Y, Li J, et al. SOCS6 promotes radiosensitivity and decreases cancer cell stemness in esophageal squamous cell carcinoma by regulating c-Kit ubiquitylation. Cancer Cell Int 2021;21:165.

21. Xue X, Liu Y, Wang Y, et al. MiR-21 and MiR-155 promote non-small cell lung cancer progression by downregulating SOCS1, SOCS6, and PTEN. Oncotarget 2016;7:84508-19. 
22. Xia Y, Wei K, Yang FM, et al. Correction: miR-1260b, mediated by YY1, activates KIT signaling by targeting SOCS6 to regulate cell proliferation and apoptosis in NSCLC. Cell Death Dis 2020;11:261.

23. Xia Y, Wei K, Yang FM, et al. miR-1260b, mediated by YY1, activates KIT signaling by targeting SOCS6 to regulate cell proliferation and apoptosis in NSCLC. Cell Death Dis 2019;10:112.

24. Bade BC, Dela Cruz CS. Lung Cancer 2020: Epidemiology, Etiology, and Prevention. Clin Chest Med 2020;41:1-24.

25. Yuan M, Huang LL, Chen JH, et al. The emerging treatment landscape of targeted therapy in non-small-cell lung cancer. Signal Transduct Target Ther 2019;4:61.

26. Bonanno L, Attili I, Pavan A, et al. Treatment strategies for locally advanced non-small cell lung cancer in elderly patients: Translating scientific evidence into clinical practice. Crit Rev Oncol Hematol 2021;163:103378.

27. Kristensen LS, Hansen TB, Veno MT, et al. Circular RNAs in cancer: opportunities and challenges in the field. Oncogene 2018;37:555-65.

28. Zhang M, Xin Y. Circular RNAs: a new frontier for cancer diagnosis and therapy. J Hematol Oncol 2018;11:21.

29. Wang S, Zhang K, Tan S, et al. Circular RNAs in body fluids as cancer biomarkers: the new frontier of liquid biopsies. Mol Cancer 2021;20:13.

30. Chen Y, Li C, Tan C, et al. Circular RNAs: a new frontier in the study of human diseases. J Med Genet 2016;53:359-65.

31. Shangguan H, Feng H, Lv D, et al. Circular RNA circSLC25A16 contributes to the glycolysis of non-smallcell lung cancer through epigenetic modification. Cell Death Dis 2020;11:437.

32. Wei S, Zheng Y, Jiang $Y$, et al. The circRNA circPTPRA

Cite this article as: $\mathrm{Ye} \mathrm{Y}, \mathrm{Wu} \mathrm{X}$, Long $\mathrm{F}$, Yue W, Wu D, Xie Y. Circular RNA_0015278 inhibits the progression of non-small cell lung cancer through regulating the microRNA 1278/SOCS6 gene axis. Ann Transl Med 2021;9(15):1255. doi: 10.21037/atm-21-3456 suppresses epithelial-mesenchymal transitioning and metastasis of NSCLC cells by sponging miR-96-5p. EBioMedicine 2019;44:182-93.

33. Hang D, Zhou J, Qin N, et al. A novel plasma circular RNA circFARSA is a potential biomarker for non-small cell lung cancer. Cancer Med 2018;7:2783-91.

34. Zhang S, Zeng X, Ding T, et al. Microarray profile of circular RNAs identifies hsa_circ_0014130 as a new circular RNA biomarker in non-small cell lung cancer. Sci Rep 2018;8:2878.

35. Yao JT, Zhao SH, Liu QP, et al. Over-expression of CircRNA_100876 in non-small cell lung cancer and its prognostic value. Pathol Res Pract 2017;213:453-6.

36. Svoronos AA, Engelman DM, Slack FJ. OncomiR or Tumor Suppressor? The Duplicity of MicroRNAs in Cancer. Cancer Res 2016;76:3666-70.

37. Du W, Hu J, Hu R, et al. circ0101675 promotes malignant process via sponging miR-1278 and upregulating WNT3A/5A in non-small cell lung cancer. J Cancer 2021;12:4209-17.

38. Zhou X, Lv L, Zhang Z, et al. LINC00294 negatively modulates cell proliferation in glioma through a neurofilament medium-mediated pathway via interacting with miR-1278. J Gene Med 2020;22:e3235.

39. Qu C, Xu Q, Lu M, et al. The involvement of suppressor of cytokine signaling 6 (SOCS6) in immune response of Chinese mitten crab Eriocheir sinensis. Fish Shellfish Immunol 2018;72:502-9.

40. Shen R, Wang Y, Wang CX, et al. MiRNA-155 mediates TAM resistance by modulating SOCS6-STAT3 signalling pathway in breast cancer. Am J Transl Res 2015;7:2115-26.

(English Language Editor: B. Meiser) 